\title{
The effect of stress-related factors on depressive state among medical residents
}

\section{Authors:}

Shoji Yokoya ${ }^{1}$,

Emiko Seo 2,3 ,

Ryoko Ogawa 2,3 ,

Ayumi Takayashiki ${ }^{1}$

Tetsuhiro Maeno 1,3

\section{Authors Note:}

1. Department of Primary Care and Medical Education, Faculty of Medicine, University of Tsukuba, Tsukuba, Ibaraki, Japan

2. Graduate School of Comprehensive Human Sciences, Faculty of Medicine, University of Tsukuba, Tsukuba, Ibaraki, Japan

3. Center for Medical Education and Training, University of Tsukuba Hospital, Tsukuba, Ibaraki, Japan

\section{Keywords}

Medical Residents; Depression; Job Stress; Sense of Coherence

\begin{abstract}
Background: Medical residents are at a high risk of depression. The National Institute for Occupational Safety and Health (NIOSH) job stress model explains that job stressors, buffer factors, and individual factors are related to worker health, including depression. This study investigated which job stressors and buffer factors influence depression in residents, independent of individual factors.
\end{abstract}

Methods: A self-administered questionnaire was distributed to second-year residents at 251 teaching hospitals. The questionnaire contained the Brief Scales for Job Stress (BSJS), the Sense of Coherence (SOC) Scale, the Center for Epidemiologic Studies Depression (CES-D) Scale, and questions on working hours and demographic characteristics. The BSJS is composed of six subscales including workload, mental workload, problems in personal relationships, job control, reward from work, and support from colleagues and superiors. The SOC includes individual factors about life stressors and health. We analyzed the relationship between depressive symptoms and these factors.

Results: Among 1,121 second-year residents who responded (64.5\% response rate), 274 (24.4\%) had depressive symptoms. In the multivariable analysis, depressive symptoms were significantly associated with SOC-13 score $(p<0.001)$, mental workload $(p<$ $0.01)$, problems in personal relationships $(p$ $<0.001)$, and reward from work $(p<0.01)$. Depressive symptoms were not significantly associated with mean number of hours worked per week.

Conclusion: Mental workload and problems in personal relationships are job stressors and reward from work is a buffer factor associated with depressive symptoms in medical residents. These findings can help residency programs support mental health of residents more effectively. 


\section{Introduction}

In medical residents, depression can result in serious, potentially life-threatening problems, such as absence from the training program, dropping out of medicine, and suicide in the worst case (Smith et al., 1986). In one meta-analysis, male physicians had a modestly higher (1.4-fold) suicide rate compared to the general population. Among female physicians, the rate was even higher (2.3-fold) (Schernhammer \& Colditz, 2004).

Beyond the effect of depression on the resident physicians themselves, it may also affect patients. Many studies show that depressed residents provide poor medical care (Beckman, 2012; Yoshino, 2007) and make more medication, procedural, or other types of medical errors (Fahrenkopf et al., 2008; Horinouchi et al., 2008; Wada et al., 2007; West et al., 2006).

Medical residents are at a high risk of depression. In a recent systematic review and meta-analysis, the summary estimate of the prevalence of depression or depressive symptoms among residents was $28.8 \%$ (95\% confidence interval [CI], 25.3\%-32.5\%), and significantly increased with baseline survey year $(0.5 \%$ per year increase) (Mata et al., 2015). Such rates are much higher than the prevalence of lifetime depression in the general population in 10 countries, which ranges from $3 \%$ to $16.9 \%$ (Andrade et al., 2003).

The depression rate is not high among medical students, but it increases after starting residency. A prospective cohort study shows that the rate of depression increased during the first year of residency, from $3.9 \%$ before internship to an average of $25.3 \%$ during the internship year (Sen et al., 2010). A prospective study in Japan reported that the rate of depression among first-year residents increased from $16.0 \%$ to $35.8 \%$ during the first two months of training (Maeno et al., 2008).

These findings suggest that a risk factor for depression is related to a work environment of a junior resident. Stress and psychosocial factors are related to the onset of depression, as well as genetic and biochemical changes (Belmaker \& Agam, 2008). Stressors for residents have been well documented in the literature. Common stressors include heavy workload, sleep deprivation, difficult patients, poor learning environment, financial concerns, information overload, and uncertainty about one's career (Levey, 2001).

The job stress model developed by the National Institute for Occupational Safety and Health (NIOSH) (NIOSH, 1999) is one of the most widely used occupational stress models. The NIOSH model explains that exposure to job stressors can have a direct influence on worker safety and health, including mental problems such as depression and burnout. Individual and buffer factors can intervene to strengthen or weaken this influence (Hurrell \& McLaney, 1988).

Antonovsky uses a concept called "sense of coherence" (SOC) to explain individual factors related to life stresses and health. SOC is defined as "a global orientation that expresses the extent to which one has a pervasive, enduring, though dynamic, feeling of confidence that one's internal and external environments are predictable and that there is a high probability that things will work out as well as can reasonably be expected" (Antonovsky \& Sagy, 1986). One review showed that SOC is an individual factor that promotes health by strengthening resilience and developing a positive subjective state of both mental and physical health (Eriksson \& Lindstrom, 2006). SOC is strongly negatively associated with anxiety, anger, burnout, demoralization, hostility, hopelessness, depression, perceived stressors, and post-traumatic stress disorder (Eriksson \& Lindstrom, 2005; 2006).

Residency program directors are expected to try to reduce job stressors and increase buffer factors. If a concrete factor that influences mental health is clearly identified, a director can support a resident effectively. However, there are few studies that clarify which specific factors influence the mental health of residents. Haoka et al. (2010) reported that workload, mental workload, and problems in personal relationships that are perceived as job stressors are negatively associated with residents' psychological well-being, and reward from work is a buffer factor that is positively associated psychological wellbeing. However, this study was conducted just after the beginning of training and the number of participants was small. Another study reported that SOC is associated with perceived stressors (Gilbar et al., 2003). No 
studies have investigated the influence of job stressors and buffer factors adjusted for SOC on depression in residents.

Therefore, we aimed to clarify which perceived job stressors and buffer factors influence depression in residents, independent of SOC.

\section{Methods}

\subsection{Participants}

Participants were second-year residents at hospitals designated as postgraduate education hospitals. In Japan, all medical residents are required to undergo 2 years of postgraduate training in several clinical medical fields, including internal medicine, emergency medicine, and primary care. They also take electives.

\subsection{Design}

Throughout Japan, there are 853 hospitals with residents, of which 251 hospitals participated in this study. In February 2011, a self-report questionnaire was distributed to participants through the residency program directors or clerks. At the time, participants were expected to complete the training two months later. Responses were voluntary and anonymous. Residents placed their completed questionnaires in sealed envelopes, which were collected by the directors or clerks.

\subsection{Questionnaire}

The questionnaires contained the Brief Scales for Job Stress (BSJS), the Japanese version of the Sense of Coherence (SOC) Scale, and the Japanese version of the Center for Epidemiologic Studies Depression (CESD) Scale. It also included objective questions about workload such as the mean number of hours worked on weekdays and weekends and number of night shifts per month.

\subsection{Center for Epidemiologic Studies Depression (CES-D) Scale}

The depressive state as a stress reaction was evaluated using the CES-D Scale. This is a 20-item questionnaire about how often one has symptoms related to depression over the past week. All items are rated on a four-point scale ranging from 0 to $3(0=$ none, $1=1-2$ days, $2=3-4$ days, and $3=\geq 5$ days). The total score can range from 0 to 60 . A cut-off score of 16 has been recommended to define a clinically significant level of depressive symptoms (Radloff, 1977). The Japanese version of the CES-D Scale has been demonstrated to have sufficiently high reliability and validity (Shima et al., 1985).

\subsection{Sense of Coherence (SOC) Scale}

Individual factors were evaluated using the 13-item version of the SOC scale (SOC-13) (Eriksson \& Lindstrom, 2005). This is a fivepoint scale ranging 1 to 5 . The SOC is composed of three factors: (1) stimuli derived from internal and external environments in the course of living are structured, predictable, and explicable (comprehensibility); (2) resources are available to meet the demands posed by these stimuli (manageability); and (3) such demands are challenges, worthy of investment and engagement

(meaningfulness) (Urakawa et al., 2012). In this study, we use the total SOC-13 score, which can range from 13 to 65 . The Japanese version of the five-point SOC-13 has been demonstrated to have sufficiently high internal consistency (Togari \& Yamazaki, 2005).

\subsection{Brief Scales for Job Stress (BSJS)}

Job stressors and buffer factors were evaluated using the BSJS. This is a 20 -item questionnaire appropriate for white-collar workers. All items are rated on a four-point scale ranging from 1 to $4(1=$ not at all, $2=a$ little, $3=$ fairly, and $4=$ extremely). Mean scores are calculated for six subscales, including workload, mental workload, problems in personal relationships, job control (job decision latitude), reward from work (job satisfaction), and support from colleagues and superiors. The first three factors are perceived job stressors, whereas the rest are buffer factors. These scales have been demonstrated to have sufficiently high internal consistency (Nishikido et al., 2000).

\subsection{Working Hours}

As in previous studies (Haoka et al., 2010; Ito et al., 2015; Taniguchi et al., 2007), mean number of hours worked per week was calculated with following equation:

Mean number of hours worked per week $=$ 5 days $\times$ (mean number of hours worked on weekdays)

+2 days $\times$ (mean number of hours worked on weekends)

$+7 / 30$ days $\times$ (number of night shifts per 
month)

$\times$ (24 hours - mean working hours on weekdays)

\subsection{Statistical Analysis}

Descriptive statistics were used to characterize sample demographics and other information. All participants were divided into those with and without depressive symptoms based on a cut-off CES-D score of 16. Univariate analysis was performed to evaluate the association between depressive symptoms and demographic factors, number of hours worked, SOC score, and the six BSJS subscale scores. The chi-square test was used for categorical variables (sex) and Student's $t$-test was used for continuous variables (other variables). Next, multivariate logistic regression analysis with forced entry for depressive symptoms was performed to adjust for confounding factors. The significance level was set at $p<0.05$. All statistical analyses were performed with IBM SPSS Statistics 23 for Windows (IBM Japan, Ltd., Tokyo, Japan). Results are reported as means $\pm S D$ unless indicated otherwise.

\subsection{Ethics}

This study was approved by the Ethics Committee of the University of Tsukuba Faculty of Medicine. All participants provided written informed consent.

\section{Results}

\subsection{Characteristics of the Study Participants}

We distributed questionnaires to 1,738 residents at 251 participating teaching hospitals, and 1,146 residents responded to the questionnaire. Replies from 1,121 residents had no missing data $(64.5 \%$ response rate) and were used for analysis. Of these, $729(65.0 \%)$ residents were male. The mean age was $28.0 \pm 3.0$ years. The mean number of hours worked per week was 71.9 \pm 15.7. Demographic factors, mean number of hours worked per week, CES-D score, SOC-13 score, and BSJS subscale scores are shown in Table 1.

\subsection{Prevalence of Depressive Symptoms}

Based on the CES-D cut-off score of 16 points, 274 (24.4\%) second-year residents had depressive symptoms (Table 1).

\subsection{Association Between Depressive Symptoms and Various Factors}

In the univariate analysis, residents with depressive symptoms worked for more hours per week than residents without depressive symptoms $(71.2 \pm 14.7$ vs $73.8 \pm$ $18.2 ; p=0.035)$. The mean SOC-13 score was significantly lower in residents with depressive symptoms $(p<0.001)$. Residents with depressive symptoms had higher mean scores for all the job stressors items and lower scores for all the buffer factor items ( $p$ $<$ 0.001). There were no significant differences in the proportion of residents with depressive symptoms by sex and age (Table 2).

\subsection{Factors Independently Associated with Depressive Symptoms}

In multivariable logistic regression analysis, depressive symptoms were significantly associated with SOC-13 score $(p<0.001)$, mental workload $(p<0.01)$, problems in personal relationships $(p<0.001)$, and reward from work $(p<0.01)$. There were no significant associations between depressive symptoms and mean number of hours worked per week, perceived workload, job control, and support from colleagues and superiors, although associations were observed in the univariate analysis. The $R^{2}$ for the multivariable model was 0.495 (Table 3).

\section{Discussion}

\subsection{Significant Factors}

This study verified that the presence of depressive symptoms in medical residents is associated with mental workload, problems in personal relationships, and reward from work, even after adjustment for the number of hours worked and the SOC-13 score. These results are consistent with findings from an earlier investigation. Haoka et al. (2004) showed that workload, mental workload, problems in personal relationships, and reward from work affect the mental health status of first-year residents.

SOC was strongly negatively associated with depression. In previous studies, a similar result was observed among medical residents (Haoka et al., 2010; Ito et al., 2015), nurses (Kikuchi et al., 2014) and factory workers (Urakawa et al., 2012). SOC might influence the strength of feelings about job stressors 
and buffering factors. In this study, it is noteworthy that concrete factors independent of SOC were identified.

\subsection{Mean Number of Hours Worked}

Interestingly, mean number of hours worked and perceived workload was not significantly associated with depressive symptoms in the multivariable analysis. Many studies (Gong et al., 2014; Kato et al., 2014; Kleppa et al., 2008; Lee et al., 2013; Virtanen et al., 2012) show that long working hours are related to depression in various occupations. On the other hand, in previous studies involving medical residents, number of hours worked per week was not linked to mental health problems (Tyssen et al., 2000), anxiety, or depression (Pereira-Lima et al., 2016).

One reason for this finding may be that the impact of the number of hours worked on mental health might have decreased. The working hours of junior residents have been adjusted downwards after the initiation of a new postgraduate residency program by order of the Japanese government. In Japan, the mean number of hours worked per week decreased to $71.9 \pm 15.7$ hours in 2011 (this study) from $86.3 \pm 16.1$ hours in 2004 (Haoka et al., 2010). Tomioka et al. (2011) showed that physicians who work 54-70 hours/week and physicians who work $<54$ hours/week have a similar prevalence of depression. However, physicians who work $>70$ hours/week have a significantly higher prevalence of depression than those who work <54 hours/week. They concluded that depression is not associated with the number of hours worked per week when it is below a certain threshold.

The present study suggests that shortening the working hours is unlikely to be an effective countermeasure against depression. Nevertheless, long working hours are not justified. Extremely long working hours may be related to depression (Tomioka et al., 2011).

\subsection{Mental Workload}

Mental workload was more strongly associated with depressive symptoms than physical workload, which is consistent with results from a study about job stress and mental health among Japanese office workers (Kawada et al., 2010). Similarly, among the various workload items, mental workload was associated with a significant adverse effect on health.

\subsection{Reward from Work}

The effort-reward imbalance (ERI) model is one theoretical model of stress (Siegrist, 1996). This model explains that the nonreciprocity of social exchanges at work (i.e., high-cost/low-gain condition at work) is associated with adverse health effects. Effort in this model corresponds to job stressor in the NIOSH model, and reward corresponds to buffer factors. Sakata et al. (2008) showed that ERI is related to depression among Japanese medical residents. Consequently, balancing effort and reward at work is important for residents' mental health. These findings support the results of the present study.

For a resident, rewards for work increase with feelings of performing meaningful work, pride, fulfillment, and satisfaction. To get these feelings, it will be necessary for residents to commit to performing medical treatments and associated duties with responsibility. When working hours are limited, resident may not sufficiently commit to their duties. In addition, as described before, limiting the number of working hours might have little direct effect on reducing depression. The present study suggests that it is more effective to reduce the mental workload than the physical workload. Therefore, residency program directors and supervisors should assess each resident's abilities and decide when it is appropriate to entrusting or assist him or her with work.

\subsection{Problems in Personal Relationships}

The present study showed that problems in personal relationships are negatively associated with depression. In Japan, medical residents rotate through many clinical departments every 1 to 6 months during training. They must build new personal relationships with supervisors, co-workers, and other healthcare staff members during each new rotation. It is important to support residents so that they become familiar with new co-workers early and build good personal relationships. 


\subsection{Limitations}

This study has some limitations. First, the cross - sectional study design prevents determining causal associations between significant independent factors and depressive symptoms. Second, only 251 of 853 hospitals $(29.4 \%)$ participated in the thus, selection bias cannot be excluded. The participating hospitals might have been more interested in resident education or health. The participating hospitals were distributed throughout Japan; there was no geographical clustering. Third, CES-D is a screening tool, and positive tests are not necessarily indicative of depressive disorders. In a validation study of the Japanese version of the CES-D (Shima et al., 1985), a cut-off of 16 had a sensitivity of $88.2 \%$ and specificity of $84.8 \%$. Fourth, it is possible that severely depressed residents could not respond to this survey. In addition, the study did not ask about disease under treatment, which may mask depressive symptoms.

\section{Conclusion}

In this study, we identified mental workload and problems in personal relationships as job stressors and reward from work as a buffering factor associated with depressive symptoms in medical residents, independent of SOC, which is an individual factor. To offer more effective support, longitudinal studies are needed to identify the causal association between factors and depression.

\section{Acknowledgments}

This work was supported by The Japan Society for the Promotion of Science KAKENHI grant number JP22590452. The authors gratefully acknowledge the residency program directors and residents who participated in this study. 


\section{References}

1. Andrade L, Caraveo-Anduaga JJ, Berglund P, et al. The epidemiology of major depressive episodes: results from the International Consortium of Psychiatric Epidemiology (ICPE) Surveys. Int J Methods Psychiatr Res. 2003; 12(1):3-21.

2. Antonovsky H, Sagy S. The development of a sense of coherence and its impact on responses to stress situations. J Soc Psychol. 1986; 126(2):213-25.

3. Beckman TJ, Reed DA, Shanafelt TD, West CP. Resident physician well-being and assessments of their knowledge and clinical performance. J Gen Intern Med. 2012; 27(3):325-30.

4. Belmaker RH, Agam G. Major depressive disorder. The New England journal of medicine. 2008; 358(1):55-68.

5. Bernburg M, Vitzthum K, Groneberg DA, Mache S. Physicians' occupational stress, depressive symptoms and work ability in relation to their working environment: a cross-sectional study of differences among medical residents with various specialties working in German hospitals. BMJ Open. 2016; 6(6):e011369.

6. Dyrbye LN, West CP, Satele D, Boone S, Tan L, Sloan J, Shanafelt TD. Burnout among U.S. medical students, residents, and early career physicians relative to the general U.S. population. Acad Med. 2014; 89(3):443-451.

7. Eriksson M, Lindstrom B. Validity of Antonovsky's sense of coherence scale: a systematic review.Journal of epidemiology and community health. 2005; 59(6):460-6.

8. Eriksson M, Lindström B. Antonovsky's sense of coherence scale and the relation with health: a systematic review. J Epidemiol Community Health. 2006; 60(5):376-81.

9. Fahrenkopf AM, Sectish TC, Barger LK, et al. Rates of medication errors among depressed and burnt out residents: prospective cohort study. BMJ (Clinical research ed). 2008; 336(7642):488-91.

10. Gilbar O. Do attitude toward cancer, sense of coherence and family high risk predict more psychological distress in women referred for a breast cancer examination? Women Health. 2003; 38(2):35-46.

11. Gong Y, Han $\mathrm{T}$, Chen $\mathrm{W}$, et al. Prevalence of anxiety and depressive symptoms and related risk factors among physicians in China: a cross-sectional study. PLoS One. 2014; 9(7):e103242.

12. Haoka T, Sasahara S, Tomotsune Y, Yoshino S, Maeno T, Matsuzaki I. The effect of stress-related factors on mental health status among resident doctors in Japan. Med Educ. 2010; 44(8):826- 834.

13. Horinouchi H, Tokuda Y, Nishimura $\mathrm{N}$, et al. Influence of Residents' Workload, Mental State and Job Satisfaction on Procedural Error: a prospective daily questionnaire-based study. General Medicine. 2008; 9(2):57-64.

14. Hurrell JJ, Jr., McLaney MA. Exposure to job stress--a new psychometric instrument. Scand J Work Environ Health. 1988; 14 Suppl 1:27-8.

15. Ito M, Seo E, Ogawa R, Sanuki M, Maeno T, Maeno T. Can we predict future depression in residents before the start of clinical training? Med Educ. 2015; 49(2):215-23.

16. Kato R, Haruyama $\mathrm{Y}$, Endo $\mathrm{M}$, Tsutsumi A, Muto T. Heavy overtime work and depressive disorder among male workers. Occup Med (Lond). 2014; 64(8):622-8.

17. Kawada $\mathrm{T}$, Ueda $\mathrm{H}$, Hayashi $\mathrm{M}$, et al. Relationship among workload, health complaints, and depressive state of workers as revealed using a questionnaire survey. Work (Reading, Mass). 2010;37(4):333-9.

18. Kikuchi Y, Nakaya M, Ikeda M, Okuzumi S, Takeda M, Nishi M. Sense of coherence and personality traits related to depressive state. Psychiatry journal. 2014; 2014:738923.

19. Kleppa E, Sanne B,Tell GS.Working overtime is associated with anxiety and depression: the Hordaland Health Study. J Occup Environ Med 2008; 50:658-666.

20. Lee KH, Kim JE, Kim YK, et al. Long working hours and emotional wellbeing in Korean manufacturing industry employees. Annals of occupational and environmental medicine. 2013; 25(1):38.

21. Levey RE. Sources of stress for residents and recommendations for programs to assist them. Acad Med. 2001; 76(2):14250.

22. Maeno T, Nakamura A, Maeno T, Ozaki M, Kimura T, Tomita E, Sasahara S, Matsuzaki I. Resident stress in the new postgraduate clinical training system. Igaku 
Kyoiku. 2008; 39(3):175-182(in Japanese). 23. Mata DA, Ramos MA, Bansal N, Khan R, Guille C, Di Angelantonio E, Sen S. Prevalence of Depression and Depressive Symptoms Among Resident Physicians: A Systematic Review and Meta-analysis. JAMA. 2015; 314(22):2373-2383.

24. NIOSH working group. Stress at work. Cincinnati, OH: National Institute for Occupational Safety and Health (NIOSH). 1999: 8.

25. Nishikido $\mathrm{N}$, Kageyama $\mathrm{T}$, Kobayashi T, Haratani T. Assessment of jobstress using a brief questionnaire: its relations to depression among male workers of an information processing company. Sangyo Seishin Hoken (Occup Ment Health). 2000; 8(2):73-82 (in Japanese).

26. Pereira-Lima K, Loureiro SR, Crippa JA. Mental health in medical residents: relationship with personal, workrelated, and sociodemographic variables. Revista brasileira de psiquiatria. 2016.

27. Radloff, L. The CES-D scale: A new self-report depression scale for research in the general population. Appl Psychol Meas. 1977; 1(3): 385-401.

28. Schernhammer ES, Colditz GA. Suicide rates among physicians: a quantitative and gender assessment (metaanalysis). The American journal of psychiatry. 2004; 161(12):2295-302.

29. Sen S, Kranzler HR, Krystal JH, et al. A prospective cohort study investigating factors associated with depression during medical internship. Arch Gen Psychiatry. 2010; 67(6):557-65.

30. Shima S, Shikano T, Kitamura T, Asai M. New Self-Rating Scale for Depression. Seishin Igaku.1985; 27(6):717723 (in Japanese).

31. Siegrist J. Adverse health effects of high-effort/low-reward conditions. J Occup Health Psychol. 1996; 1(1):27-41.

32. Smith JW, Denny WF, Witzke DB. Emotional impairment in internal medicine house staff. Results of a national survey. JAMA : the journal of the American Medical Association. 1986; 255(9):1155-8.

33. Taniguchi K, Sasahara S, Maeno T, Yoshino S, Tomotsune Y, Tomita E, Usami K, Haoka T, Doki S, Matsuzaki I. Working environment, job stress factor and mental health among Japanese medical residents and supervisors. Journal of Physical Fitness, Nutrition and Immunology. 2007; 17:223229.

34. Togari T, Yamazaki Y. Examination of the reliability and factor validity of 13item five-point version Sense of Coherence Scale. Minzoku Eisei. 2005; 71(4):168182(in Japanese).

35. Tomioka K, Morita N, Saeki K, Okamoto N, Kurumatani N. Working hours, occupational stress and depression among physicians. Occup Med (Lond). 2011; 61(3):163-70.

36. Tyssen R, Vaglum P, Gronvold NT, Ekeberg O. The impact of job stress and working conditions on mental health problems among junior house officers. A nationwide Norwegian prospective cohort study. Med Educ. 2000; 34(5):374-84.

37. Urakawa K, Yokoyama K, Itoh H. Sense of coherence is associated with reduced psychological responses to job stressors among Japanese factory workers. BMC Res Notes. 2012; 5:247.

38. Virtanen M, Stansfeld SA, Fuhrer R, Ferrie JE, Kivimaki M. Overtime work as a predictor of major depressive episode: a 5year follow-up of the Whitehall II study. PLoS One. 2012; 7(1):e30719.

39. Wada K, Sakata Y, Fujino Y, Yoshikawa T, Tanaka K, Miyajima E, Aizawa Y. The association of needlestick injury with depressive symptoms among first-year medical residents in Japan. Ind Health. 2007; 45(6):750-755.

40. West CP, Huschka MM, Novotny PJ, et al. Association of perceived medical errors with resident distress and empathy: a prospective longitudinal study. JAMA : the journal of the American Medical Association. 2006; 296(9):1071-8.

41. Yoshino S, Sasahara S, Maeno T, et al. Relationship between mental health of Japanese residents and the quality of medical service. Journal of Physical Fitness, Nutrition and Immunology. 2007; 17(1):311. 


\section{Table 1}

\section{Basic Characteristics of the Study Participants}

$\begin{array}{lclll}\text { Sex, } n(\%) & & & & \\ \text { Male } & 729 & (65.3 \%) & \\ \text { Female } & 392 & (34.7 \%) & \\ \text { Age, years, mean } \pm S D \text { (range) } & 28.0 & \pm & 3.0 & (25-63) \\ \text { Mean number of hours worked per week, mean } \pm S D \text { (range) } & 71.9 & \pm & 15.7 & (22-150) \\ & & & & \\ & 11.0 & \pm & 8.3 & (0-60) \\ \text { CES-D score, mean } \pm S D \text { (possible range) } & 43.4 & \pm & 7.9 & (13-65) \\ \text { SOC-13 score, mean } \pm S D \text { (possible range) } & & & \\ \text { BSJS score, mean } \pm S D \text { (possible range) } & 1.87 & \pm & 0.68 & (1-4) \\ \text { Workload } & 1.93 & \pm & 0.65 & (1-4) \\ \text { Mental workload } & 1.67 & \pm & 0.64 & (1-4) \\ \text { Problems in personal relationships } & 2.47 & \pm & 0.64 & (1-4) \\ \text { Job control } & 2.89 & \pm & 0.73 & (1-4) \\ \text { Reward from work } & 2.92 & \pm & 0.64 & (1-4) \\ \text { Support from colleagues and superiors } & & & \end{array}$

$S D=$ standard deviation

CES-D = Center for Epidemiologic Studies Depression; $\mathrm{SOC}=$ Sense of Coherence BSJS = Brief Scales for Job Stress 
Table 2

Univariate Analysis of Factors Associated with Depressive Symptoms Among Second-year Residents

\begin{tabular}{lcrlc}
\hline & $\begin{array}{c}\text { CES-D score }<16 \\
n=847\end{array}$ & $\begin{array}{c}\text { CES-D score } \geq 16 \\
n=274\end{array}$ & $p^{\text {a }}$ \\
& & & & \\
\hline Sex & $553(75.9 \%)$ & 176 & $(24.1 \%)$ & \\
Male, $n(\%)$ & $294 \quad(75.0 \%)$ & $98 \quad(25.0 \%)$ & 0.77 \\
Female, $n(\%)$ & & & \\
& & & \\
& Mean value $\pm S D$ & Mean value $\pm S D$ & \\
Age & $27.9 \pm 2.8$ & $28.2 \pm 3.6$ & 0.22 \\
Mean number of hours worked per week & $71.2 \pm 14.7$ & $73.8 \pm 18.2$ & 0.035 \\
SOC-13 score & $45.7 \pm 7.0$ & $36.3 \pm 5.7$ & $<0.001$ \\
BSJS score & & & & \\
$\quad$ Workload & $1.77 \pm 0.62$ & $2.19 \pm 0.74$ & $<0.001$ \\
Mental workload & $1.80 \pm 0.60$ & $2.33 \pm 0.65$ & $<0.001$ \\
Problems in personal relationships & $1.53 \pm 0.56$ & $2.11 \pm 0.69$ & $<0.001$ \\
Job control & $2.54 \pm 0.62$ & $2.27 \pm 0.67$ & $<0.001$ \\
$\quad$ Reward from work & $3.01 \pm 0.69$ & $2.51 \pm 0.72$ & $<0.001$ \\
Support from colleagues and superiors & $3.01 \pm 0.63$ & $2.65 \pm 0.60$ & $<0.001$ \\
\hline
\end{tabular}

$S D=$ standard deviation

${ }^{a}$ Sex: chi-square test; other: Student's $t$-test

CES-D = Center for Epidemiologic Studies Depression; SOC = Sense of Coherence BSJS $=$ Brief Scales for Job Stress 
Table 3

Logistic Regression Analysis of Depressive Symptoms and Working Hours, Sense of Coherence, Job Stressors and Buffer Factors Among Second-Year Residents

\begin{tabular}{lccc}
\hline Variable & OR & $(95 \% \mathrm{CI})$ & $p$ \\
\hline Sex & & & \\
Male(referent) & & & 0.89 \\
Female & 1.03 & $(0.71-1.48)$ & 0.79 \\
Mean number of hours worked per week & 1.00 & $(0.99-1.01)$ & $<0.001$ \\
SOC-13 score & 0.83 & $(0.80-0.86)$ & 0.62 \\
BSJS score & & & $<0.01$ \\
Workload & 1.10 & $(0.76-1.58)$ & $<0.001$ \\
Mental workload & 1.86 & $(1.26-2.75)$ & 0.46 \\
Problems in personal relationships & 1.92 & $(1.43-2.58)$ & 0.01 \\
Job control & 0.89 & $(0.65-1.21)$ & 0.32 \\
Reward from work & 0.59 & $(0.44-0.80)$ & \\
Support from colleagues and superiors & 0.84 & $(0.59-1.19)$ & \\
\hline
\end{tabular}

$n=1121 ;$ Nagelkerke's $R^{2}=0.495$

$\mathrm{OR}=$ odds ratio; $\mathrm{CI}=$ confidence interval

CES-D = Center for Epidemiologic Studies Depression; SOC = Sense of Coherence BSJS $=$ Brief Scales for Job Stress 